\title{
Determination of volatile alkylpyrazines in microbial samples using gas chromatography-mass spectrometry coupled with head space-solid phase microextraction
}

\author{
So Hyeon Jeon ${ }^{1,2}$, Jeoung Hwa Shin ${ }^{3}$, Yong Pyo Kim²,4 and Yun Gyong Ahn ${ }^{1 *}$
}

\begin{abstract}
Background: Determination of volatile alkylpyrazines (VAPS) in microbial samples is needed for the understanding of microbial metabolism. An analytical method for screening and quantification of six volatile alkylpyrazines was developed and successfully applied to the real microbial samples.

Methods: Headspace-solid phase microextraction (HS-SPME) which is a simple extraction simultaneously carrying out clean-up and pre-concentration of VAPs in microbial samples was adopted. GC-TOF/MS with CombiPal SPME autosampler was used for fast screening of microbial samples and the optimization of HS-SPME condition. Separation and quantification of six VAPs that have the potential to be detected in microbial samples using quadrupole GC/MS in chemical ionization (Cl) mode were carried out.
\end{abstract}

Results: The optimal extraction conditions for HS-SPME at $50{ }^{\circ} \mathrm{C}$ and 50 min were investigated. To eliminate matrix effects, DB-WAX column was more appropriate to separate each alkylpyrazines from the interferences than DB-5MS column. Chemical ionization provided enhanced confirmation from adduct ions $\left([\mathrm{M}+29]^{+}\right.$and $\left.[\mathrm{M}+41]^{+}\right)$of methane reagent gas as well as increasing signal intensity of molecular ions for quantitative analysis. Linear calibration curves of six alkylpyrazines were obtained within a range between 1 and $50 \mathrm{ng} / \mathrm{g}$.

Conclusions: This proposed method was successfully applied to the microbial samples. 2-Methylpyrazine and 2,5-dimethylpyrazine were determined in the level of $6.0 \sim 34.0 \mathrm{ng} / \mathrm{g}$.

Keywords: Volatile alkylpyrazines (VAPs), Microbial samples, Gas chromatography/mass spectrometry (GC/MS) , Head space-solid phase microextraction (HS-SPME), Chemical ionization (CI)

\section{Introduction}

Pyrazines are heterocyclic aromatic organic compounds containing nitrogen atoms in the 1- and 4-position of the aromatic ring. Pyrazines are found in a wide variety of foods processed during dry heating processes (Muller and Rappert 2010). They are also synthesized and degraded by microorganisms during their primary or secondary metabolism which occurs by Maillard reaction and Strecker's degradation (Hwang et al. 1994; Shu

\footnotetext{
* Correspondence: ygahn@kbsi.re.kr

${ }^{1}$ Western Seoul Center, Korea Basic Science Institute, Seoul, South Korea Full list of author information is available at the end of the article
}

1999). It is generally known that pyrazines are not produced by enzymatic reactions in microorganisms, but are thought to arise from the non-enzymatic condensation between the precursors of amino acids (Koehler et al. 1969; Muller and Rappert 2010). The lower methyl and ethyl pyrazines were formed non-enzymatically by amination of acyloins leading to aminocarbonyl compounds followed by condensation (Rizzi 1988). Diverse alkylated pyrazines were formed by reaction of acyloins with methyl, ethyl, and propyl substitutions leading to different alkylated pyrazines (Rajini et al. 2011; Schulz and Dickschat 2007). They are responsible for different 
flavors according to the nature of the alkyl substituents. Therefore, the understanding synthesized pyrazines from microbial metabolism for finding key flavors is very important. However, analysis of pyrazines in food has been investigated actively, while few studies have been performed in analytical methods of pyrazines from microbial metabolism. Furthermore, pyrazines produced by microorganisms can be found in low quantities in culture media (Beck et al. 2003; Dickschat et al. 2010; Nawrath et al. 2010), and the bottleneck of analysis has been the difficulty of sample preparation due to the high solubility and volatility of pyrazines in microbial samples. Conventional techniques have been used previously such as headspace and purge and trap. Headspace extraction used for volatile analytes in solid or liquid samples is preferable technique for a quality control but the sensitivity is limited (Pawliszyn 2000). The purge and trap technique is more sensitive, while the complicated switching valves and ghost peaks caused by decomposition of the trapping materials during thermal desorption are main disadvantages. Recently, SPME which was invented by Pawliszyn in 1989 has been applied to analysis in various fields, such as environmental chemistry, forensic chemistry, pharmaceutical, food, beverage, and flavors (Pawliszyn 2000; Theodoridis et al. 2000). The advantages of SPME over the previous techniques are that it is a very simple and efficient sample preparation method as sampling, extraction, and concentration can be combined into a single solvent-free step. Other advantages of SPME are that it can use small sample volumes (Risticevic et al. 2010). As a diffusion micro sampling tool, solid porous coating fibers can adsorb analytes from a sample by immersion or headspace extraction. Generally, direct immersion is not suitable for complex media and may damage fiber in direct contact with dirty matrix (Pawliszyn 2000). For these reasons, headspace-solid phase microextraction (HS-SPME) was adopted to determine volatile alkylpyrazines (VAPs) in microbial samples as a sampling technique in this study. Meanwhile, the nitrogen-phosphorus detector (NPD) or flame ionization detector (FID) has been used to detect VAPs but various types of mass spectrometric detectors have been employed increasingly due to the availability for identification and quantification of VAPs (Harris et al. 1987; Sala et al. 2002; Clery et al. 2006).

In the present work, we report a suitable analytical method for screening and quantification of six VAPs in microbial samples. GC time of flight mass spectrometer (GC-TOFMS) has the capability of very fast data acquisition (up to 500 spectra/second) to provide a full mass spectrum for each chromatographic point (Bergknut et al. 2006). Whereas GC quadrupole mass spectrometer which is conventional technique for quantitative analysis has lower sample rate than GC-TOFMS. However, it has advantage of high sensitivity to deliver more accurate results in the selected ion monitoring (SIM) and detailed spectrum results of each molecular ions in chemical ionization $(\mathrm{CI})$ mode. In this paper, GC-TOFMS for the screening of VAPs in microbial sample and GC/CI-MS for quantitative analysis of six VAPs that have the potential to be detected in the sample were applied. The practical applicability of this analytical method could be successfully demonstrated in the Acetobacter pomorum, kind of microbial samples.

\section{Methods}

\section{Chemicals and reagents}

Six VAPs (2-methylpyrazine (MP), 2,5-dimethylpyrazine (DMP), 2-ethylpyrazine (EP), 2-ethyl-3,5-dimethylpyrazine (EDMP), 2-isopropyl-3-methoxy pyrazine (IPMP), and 2-ethoxy-3-ethylpyrazine (EEP); see Table 1 for their full chemical names and information) for GC/MS analysis were purchased from Sigma-Aldrich (St. Louis, $\mathrm{MO}$, USA) and stock solution as a mixture at a concentration of $1000 \mu \mathrm{g} / \mathrm{L}$ in methanol. Deuterium-labeled internal standards of n-nitrosodipropylamine- $\mathrm{d}_{14}$ (NDPA$\mathrm{d}_{14}$ ) was purchased from $\mathrm{C} / \mathrm{D} / \mathrm{N}$ isotopes (Pointe-Claire, QC, Canada). Working standard solutions to check the calibration were prepared in the range of 1 to $50 \mathrm{ng} / \mathrm{g}$ and then stored at $-20{ }^{\circ} \mathrm{C}$ prior to use. Organic solvents

Table 1 Detailed information for six volatile alkylpyrazines and internal standard

\begin{tabular}{|c|c|c|c|c|c|c|c|}
\hline \multirow[t]{2}{*}{ Compound } & \multirow[t]{2}{*}{ Abbreviation } & \multirow[t]{2}{*}{ CAS } & \multirow[t]{2}{*}{ Formula } & \multirow[t]{2}{*}{ B.P. $\left({ }^{\circ} \mathrm{C}\right)$} & \multirow[t]{2}{*}{ M.W. } & \multicolumn{2}{|c|}{$\begin{array}{l}\text { GC retention time } \\
\text { (min) }\end{array}$} \\
\hline & & & & & & DB-5MS & DW-WAX \\
\hline 2-Methylpyrazine & MP & 109-08-0 & $\mathrm{C}_{5} \mathrm{H}_{6} \mathrm{~N}_{2}$ & 135 & 94 & 4.4 & 6.5 \\
\hline 2,5-Dimethylpyrazine & DMP & $123-32-0$ & $\mathrm{C}_{6} \mathrm{H}_{8} \mathrm{~N}_{2}$ & 155 & 108 & 5.4 & 6.9 \\
\hline 2-Ethylpyrazine & EP & $13925-00-3$ & $\mathrm{C}_{6} \mathrm{H}_{8} \mathrm{~N}_{2}$ & $152 \sim 153$ & 108 & 5.4 & 7.0 \\
\hline 2-Ethyl-3,5-dimethylpyrazine & EDMP & $27043-05-6$ & $\mathrm{C}_{8} \mathrm{H}_{12} \mathrm{~N}_{2} \mathrm{O}$ & $180 \sim 181$ & 136 & 6.9 & 7.8 \\
\hline 2-Isopropyl-3-methoxypyrazine & IPMP & $25773-40-4$ & $\mathrm{C}_{8} \mathrm{H}_{12} \mathrm{~N}_{2} \mathrm{O}$ & $120 \sim 125$ & 152 & 7.0 & 7.7 \\
\hline 2-Ethoxy-3-ethylpyrazine & EEP & $35243-43-7$ & $\mathrm{C}_{8} \mathrm{H}_{12} \mathrm{~N}_{2} \mathrm{O}$ & 195 197 & 152 & 7.2 & 7.9 \\
\hline n-Nitrosodi-n-propylamine-d ${ }_{14}$ & NDPA-d 14 & $93951-96-3$ & $\mathrm{C}_{6} \mathrm{D}_{14} \mathrm{~N}_{2} \mathrm{O}$ & 206 & 144 & 7.0 & 8.3 \\
\hline
\end{tabular}


(methanol, distilled water) of GC analysis grade were purchased from Burdick \& Jackson (Philipsburg, NJ, USA).

\section{Preparation of samples}

The preparation of microorganisms was given from school of biological science in Seoul National University. A. pomorum, species of microorganisms, was isolated from the intestine of Drosophila and described in detail by Shin et al. (2011). Homogenized sample of $1 \mathrm{~mL}$ was spiked with $100 \mathrm{ng}$ of the internal standard in the $4 \mathrm{~mL}$ headspace vial for quantitative analysis.

\section{Instrumentation}

To optimize extraction time and temperature, HS-SPME sampling was performed using an Agilent GC (Wilmington, Delaware, USA)-Pegasus 4D TOFMS (St. Joseph, Michigan, USA) equipped with a combiPAL SPME autosampler (CTC Analytics, Zurich, Switzerland). A $75 \mu \mathrm{m}$ carboxen/polydimethylsiloxane (CAR/PDMS) fiber which has been demonstrated as an optimal fiber for the alkylpyrazines analysis in the previous studies (de Oliveira et al. 2004) was purchased from Supelco (Bellefonte, PA, USA) and used. For quantitative analysis, GCCI/MS was performed by an Agilent $7890 \mathrm{~B}$ gas chromatograph, equipped with a 7693 mass selective detector single quadrupole mass spectrometer system (Palo Alto, CA, USA). Chromatographic separation was achieved using a DB-WAX or DB-5MS $(30 \mathrm{~m} \times 0.25 \mathrm{~mm}$ I.D; $0.25-\mu \mathrm{m}$ film thickness) from J\&W Scientific capillary column (Santa Clara, CA, USA) along with a Siltek SPME splitless liner $0.75 \mathrm{~mm}$ i.d. from Supelco (Bellefonte, PA, USA). A $2 \mathrm{~mL}$ of sample in 10-mL capped glass vial was salt-enriched medium and agitated during the extraction. In the case of manual injection mode, the headspace volume is allowed to be minimized in a $4 \mathrm{~mL}$ vial instead of $10-\mathrm{mL}$ vial for autosampler. By minimizing the headspace volume, the amount of analytes adsorbed onto the fiber in the gas phase can be maximized (Yang and Peppard 1994). The setting on the manual SPME is described in more detail in our previous study (Choi et al. 2016). The GC oven temperature was maintained at $40{ }^{\circ} \mathrm{C}$ for $2 \mathrm{~min}$ and then reached to 320 at $10{ }^{\circ} \mathrm{C}$ per min for DB-5MS and to limited temperature, $240{ }^{\circ} \mathrm{C}$ for DB-WAX column. The GC method injection port temperature was set to $250{ }^{\circ} \mathrm{C}$. High-speed Pegasus TOFMS, not equipped for $\mathrm{CI}$, was operated in electron impact (EI) mode at $70 \mathrm{eV}$ electron energy. The MS transfer line temperature was set to $250{ }^{\circ} \mathrm{C}$. The MS mass range was set at 50 550 amu with an acquisition rate of 10 spectra per second. The ion source chamber was held at $200{ }^{\circ} \mathrm{C}$, and the detector voltage was set to $1850 \mathrm{~V}$. ChromaTOF software was used for all acquisition control and data processing. The quadrupole mass spectrometer was able to use both EI and $\mathrm{CI}$ mode. For this, ion source temperature was $250{ }^{\circ} \mathrm{C}$ and $\mathrm{CI}$ reagent gas was methane (flow, $20 \%$ ). Acquisition was carried out in both scan and SIM mode.

\section{Results and discussion}

Screening of alkylpyrazines in microbial sample

The GC-TOFMS was used for screening of the compounds in microorganisms. The advantage of this types of mass spectrometer offers very fast spectral acquisition rates, allowing the separation of a substantial number of compounds. SPME integrates sampling, extraction, and concentration in one step regarding the large data sets of volatile organic compounds. High-speed TOFMS is capable of providing the multi-residue screening and confirmation of positive findings for the target analytes than quadrupole mass analyzer which has moderate scan speeds of $5 \sim 15 \mathrm{scan} / \mathrm{s}$. In order to obtain broad coverage of volatile compounds in microorganisms, $2 \mathrm{~mL}$ of sample was analyzed by GC-TOFMS with CTC analytics CombiPal autosampler with SPME. The peak list was generated by ChromaTOF, and about 100 peaks were identified by matching over $80 \%$ against the NISTMS library search. VAPs were positively found, but unsuccessful library search has occurred when the concentration of analyte was low level and co-eluting the matrix interference. Even though mass spectral deconvolution, the process of computationally separating co-eluting analytes creating a pure spectrum for each analyte, was processed by ChromaTOF software, quantification of ions of alkylpyrazines in the TOF mass analyzer could not well defined in comparison to SIM in the quadrupole mass analyzer. Furthermore, chemical ionization quadrupole mass spectrometer which is available switching between EI and CI techniques was suited better than EI-fast TOFMS for the quantification of target alkylpyrazines. Thus, the analytical strategy in this paper was focused first on the optimization of extraction parameters and fast screening of target analytes by EI-fast TOFMS with CombiPal SPME autosampler. Second, the separation and quantification of target alkylpyrazines were explored by CI-SIM quadrupole MS.

\section{Optimization of HS-SPME}

The main principle of headspace SPME is partition equilibrium of three phase systems such as fiber coating, headspace, and sample (Zhang and Pawliszyn 1993). An absorbed amount of analytes on fiber coating and sensitivity depend on sorption kinetics and partition coefficient between fiber coating and a sample (Yang and Peppard 1994). Therefore, parameters that effect partition coefficient and sorption rate also influence on an absorbed amount of analytes, reproducibility, and sensitivity in headspace SPME. The 
partition equilibrium in headspace SPME system depends on temperature, extraction time, headspace volume, sample volume, agitation in sample, initial concentration of sample, and type and uniformity of the matrix (Zhang and Pawliszyn 1993; Yang and Peppard 1994; Wampler 1997). In this study, the optimum conditions of extraction time and temperature were investigated on a $75 \mu \mathrm{m}$ PDMS/ carboxen fiber; $2 \mathrm{~mL}$ of the matrix sample spiked with target alkylpyrazines in $10 \mathrm{~mL}$ commercially available autosampler vial was used at the concentration of $500 \mathrm{ng} / \mathrm{mL}$. Extraction temperature of sample has significant effects on kinetic process (Pawliszyn 2000) because it determines vapor pressure of analytes in the sample. The extraction efficiencies of target pyrazines in microbial sample were observed in various extraction temperatures $(40,50,60,70$, and $80{ }^{\circ} \mathrm{C}$ ). MP showed relatively small change of extraction efficiency under given conditions. DMP and EP showed decreasing trends as temperature of sample increased. They showed high extraction efficiency at $50{ }^{\circ} \mathrm{C}$ and significantly decreased over $50{ }^{\circ} \mathrm{C}$ as shown in Fig. 1. Therefore, $50{ }^{\circ} \mathrm{C}$ was most adequate temperature for extraction of VAPs in microbial samples. Extraction time is usually determined by an equilibrium partitioning of analytes from the aqueous phase to the SPME fiber. Extraction times from 30 to $50 \mathrm{~min}$ were investigated to determine time taken to reach equilibrium for target analytes. As seen in Fig. 2, equilibrium was not reached at $50 \mathrm{~min}$ even though the sample was prepared under the sufficient agitation and salt content. In this study, an extraction time of $50 \mathrm{~min}$ was chosen under the non-equilibrium extraction condition. When using non-equilibrium extraction, care should be taken consistently to extract target analytes under

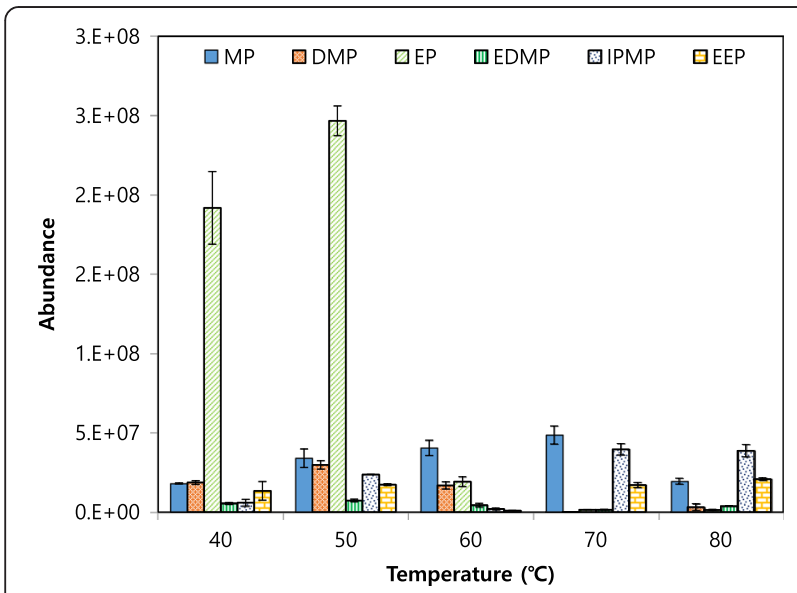

Fig. 1 Comparison of extraction efficiency of each alkylpyraizne at different temperatures of $40,50,60,70$, and $80^{\circ} \mathrm{C}$

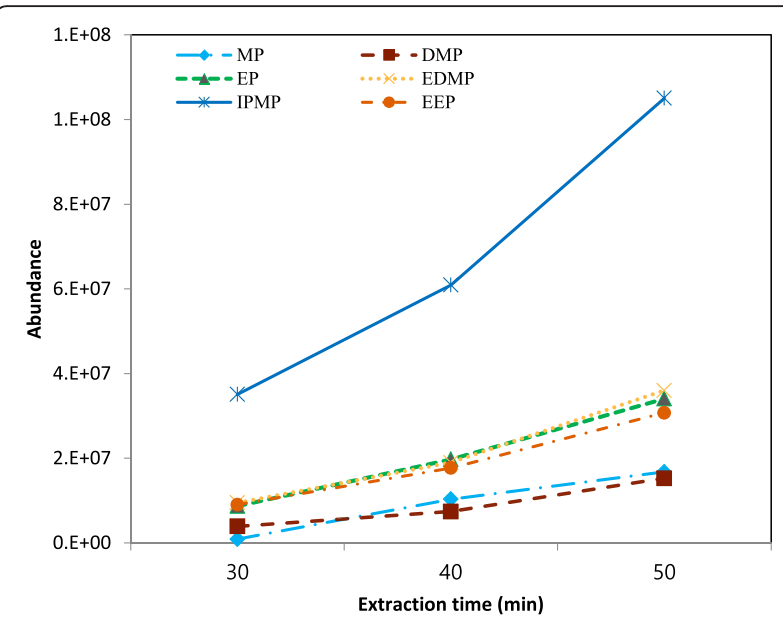

Fig. 2 Response of each alkylpyrazine according to the extraction times

the same condition for the quantitative analysis. This problem is also aided by the use of an extraction surrogate as a labeled standard to account for slight variations between sample runs (Grebel et al. 2006). For this reason, the labeled standard was considered but VAPs have limitation of the unavailability of labeled standards. It is not widely used due to the high cost associated with the purchase or synthesis of the isotopically labeled standards (Fang and Cadwallader 2013). Instead, n-nitrosodipropylamine- $\mathrm{d}_{14}$ which is a nitro compound similar to target analytes being extracted was used to precisely control the extraction conditions for further quantitative analysis.

\section{Chromatographic separation and detection of alkylpyrazines}

After extraction, SPME fiber is desorbed in the GC injection port and then large amounts of matrix interferences as well as target analytes are eluted to a capillary column. Therefore, the chromatographic separation and the detection selectivity are crucial for the quantitative analysis. Separation in the gas chromatography depends on several factors such as column length, column diameter, stationary phase, temperature programming, carrier gas velocity, pressure, and void time (Harris 2010). Among them, a sort of stationary phase in column influences on retention time and separation of analytes in a sample, directly. The DB-5MS stationary phase column which is commonly used because of the stability and robustness. The results were compared with a DB-5MS column and a DB-WAX in a standard mixture as shown in Fig. 3. Three analytes of VAPs were well separated on the DB-5MS column, but the rest of the analytes were partially separated. In comparison, all analytes were completely separated on the DB-WAX in some type of 


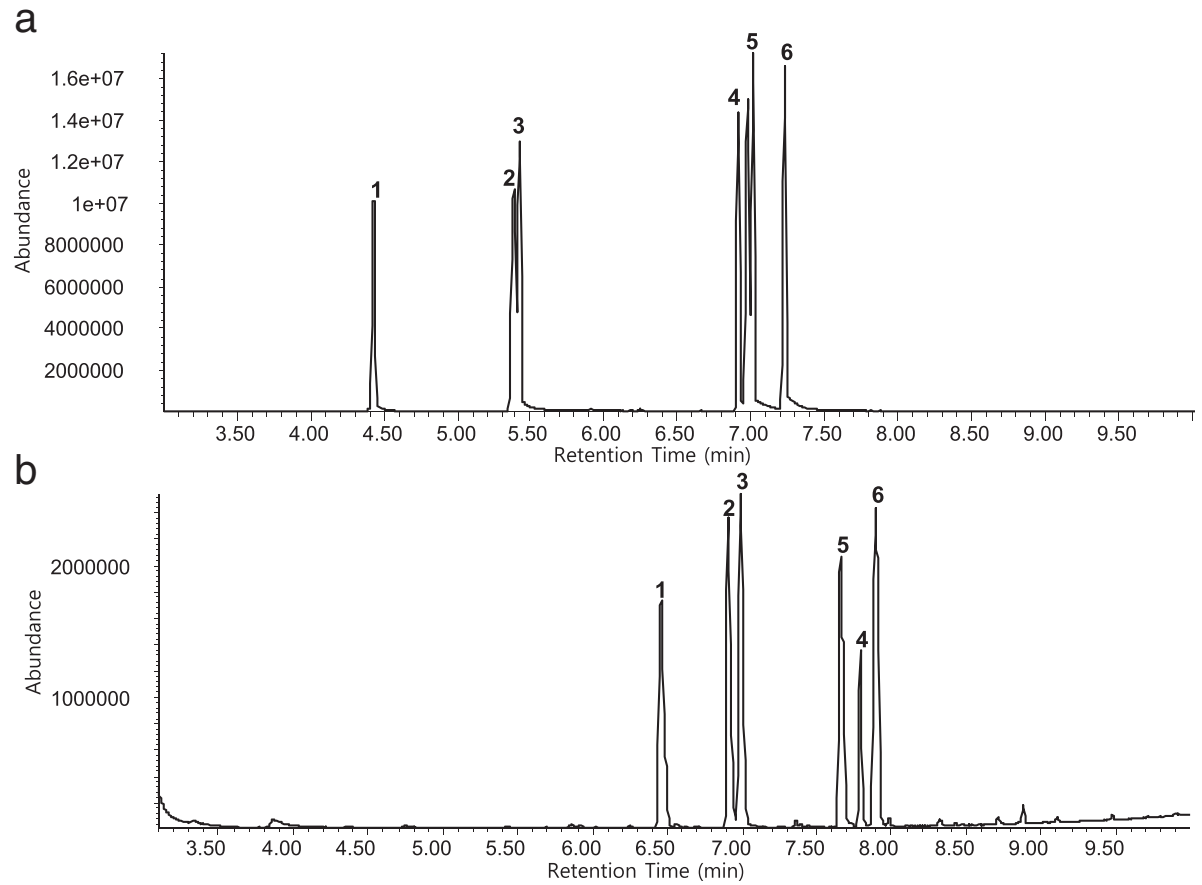

Fig. 3 The chromatograms of VAPs in the standard mixture analyzed on the DB-5MS (a) and DB-WAX (b) columns. Peak identities as follows: 1. $M P$, 2. DMP, 3. EP, 4. EDMP, 5. IPMP, 6. EEP

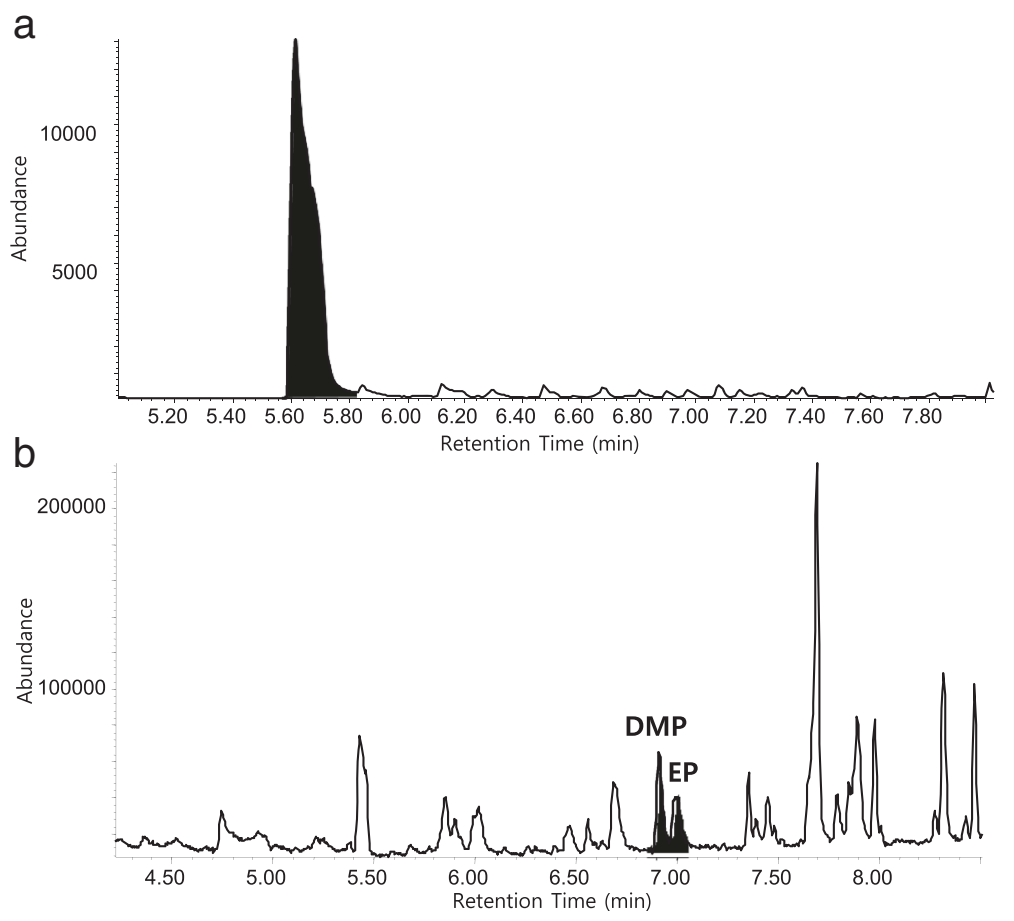

Fig. 4 SIM and scan chromatograms of 2,5-dimethylpyrazines (DMP) and 2-ethylpyrazine (EP) in the microbial sample on the DB-5MS (a) and $\mathrm{DB}-\mathrm{WAX}(\mathbf{b})$ columns 


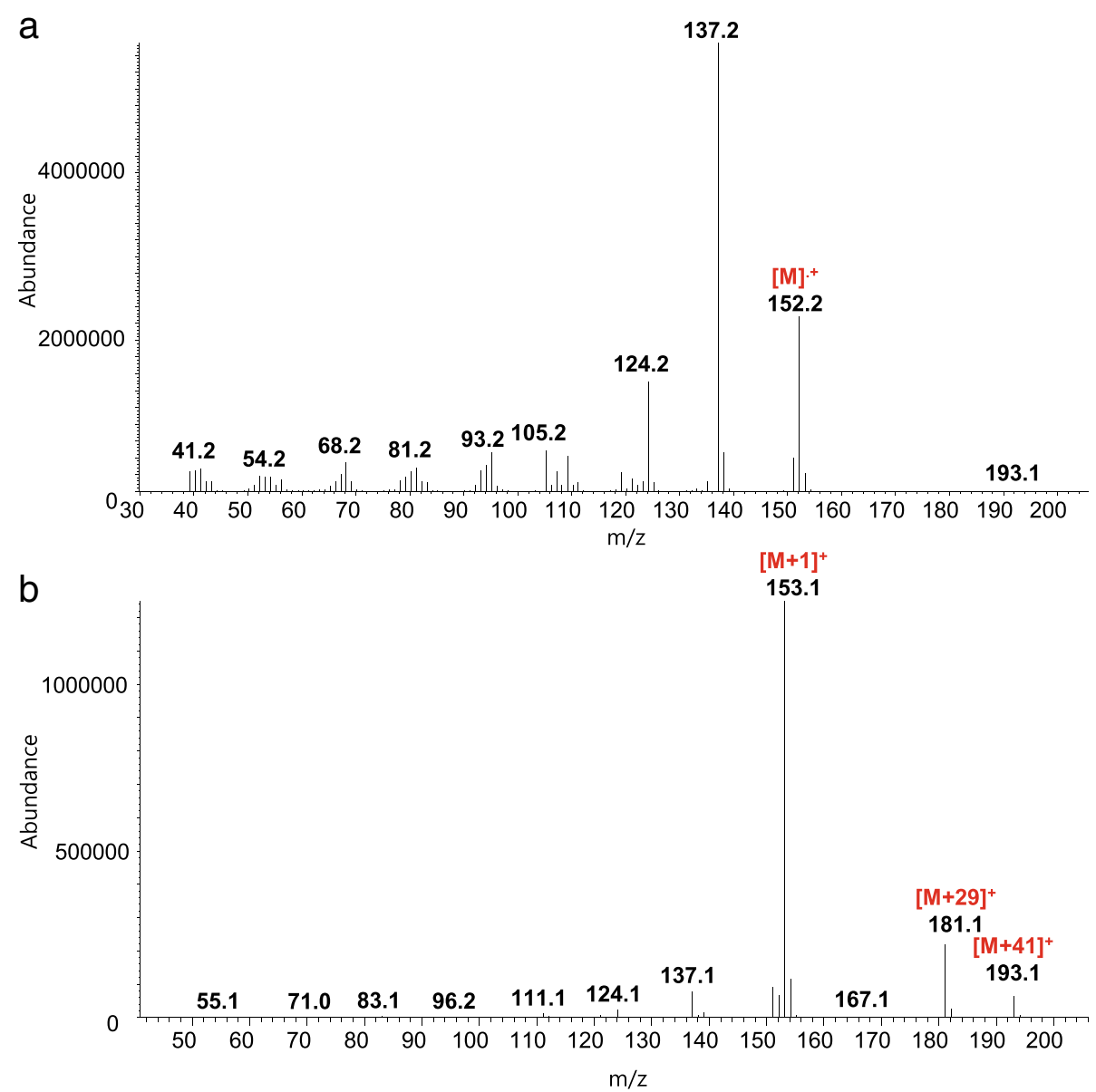

Fig. 5 Mass spectrum of 2-isopropyl-3-methoxy pyrazine (IPMP) in El (a) and $\mathrm{Cl}$ (b) modes

polyethylene glycols (PEG) column. The elution order of EDMP and IPMP from two columns was reversed and represented in Table 1 . As a result of chromatographic separation in the microbial sample, DMP was not separated from the sample matrix on the DB-5MS column but clearly separated on the DB-WAX. Figure 4 showed SIM and scan chromatograms obtained by DB-5MS and DB-WAX columns when applied to a real microbial sample. The DB-WAX column was able to separate DMP from the sample matrix even in scanning mode (Fig. 4b) in comparison with DB-5MS even in selected ion monitoring mode (Fig. 4a). For the detection selectivity of VAPs, CI as a soft ionization technique was compared with EI method. The higher signal intensity of molecular ions for VAPs in CI was provided than EI. Figure 5 showed the typical mass spectrum of IPMP in $\mathrm{EI}$ and CI methods. The molecular ion of IPMP in CI mass spectrum was clearly confirmed by detection of additional methane reagent gas adducts $\left([\mathrm{M}+29]^{+}\right.$for $\left[\mathrm{M}+\mathrm{C}_{2} \mathrm{H}_{5}\right]^{+}$and $[\mathrm{M}+41]^{+}$for $\left.\left[\mathrm{M}+\mathrm{C}_{3} \mathrm{H}_{5}\right]^{+}\right)$as well as higher intensity than EI. It suggested the appropriate

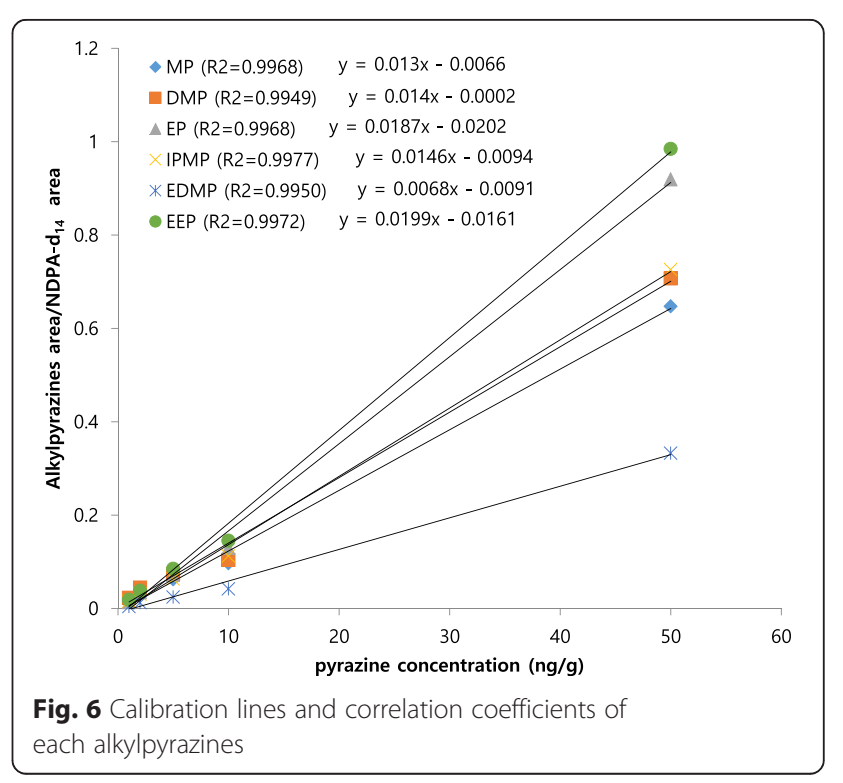



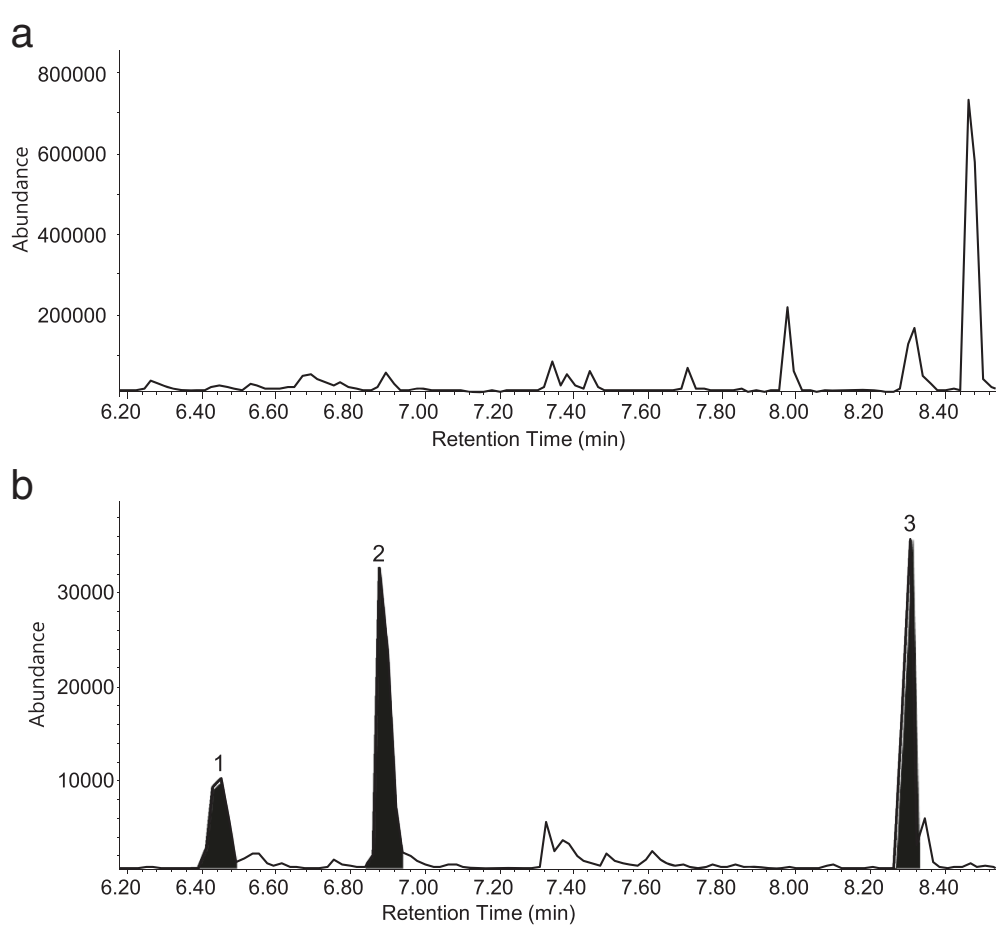

Fig. 7 Scan (a) and SIM (b) chromatograms of VAFs in a positive microbial sample. Peak identities as follows: 1 MP (6.0 ng/g), 2 DMP (12.1 ng/g), 3 NDPA $^{-d_{14}}$ (IS)

DB-WAX column and CI method for separation and detection of six VAPs in microbial samples.

\section{Quantification in real samples}

For the quantitative analysis of VAPs in real microbial samples, the calibration curves were generated within a range between 1 and $50 \mathrm{ng} / \mathrm{g}$ using a least-squares linear regression analysis. These were calculated from the ratio of the peak area of target VAPs to the peak area of deuterium-labeled internal standard, $n$-nitrosodipropylamine- $\mathrm{d}_{14}$. The correlation coefficients of six VAPs were above 0.99 as shown in Fig. 6. With the optimized method, the determination of six VAPs in the $A$. pomorum, a kind of microbial samples was carried out by the labeled internal standard method. A typical chromatogram of target alkylpyrazines extracted from the $A$. pomorum sample is given in Fig. 7. MP and DMP were mainly detected in the sample, and no significant interferences were observed in the selected ion chromatogram. Their concentration ranges were between 6.0 and $34.0 \mathrm{ng} / \mathrm{g}$.

\section{Conclusion}

In the present study, GC-TOFMS coupled with headspace SPME was used for fast screening of alkylpyrazines in microbial samples. Six volatile alkylpyrazines that have the potential to be detected in the sample were selected and then the method for the separation and detection was developed. For the chromatographic separation, DB-WAX column was more effective to separate 2,5-dimethylpyrazine from the matrix interferences than the most common stationary phase, DB-5MS column. To increase signal intensity of molecular ions for alkylpyrazines, chemical ionization was adopted for quantitative analysis. It provided enhanced confirmation from adduct ions $\left([\mathrm{M}+29]^{+}\right.$and $\left.[\mathrm{M}+41]^{+}\right)$of methane reagent gas. This method can be applied to bioflavour and its related research fields for the identification and quantification of volatile alkylpyrazines.

\section{Acknowledgements}

This work was supported by the Korea Basic Science Institute grant (C36705). We thank Professor Lee in Seoul National University for providing the samples.

\section{Authors' contributions}

YGA designed the study. SHJ and JHS carried out the GC-MS analysis. Supervision of experimental work and analysis of results were done by YPK and YGA. SHJ and YGA contributed to the draft version of the manuscript. All the authors read and approved the final manuscript.

\section{Competing interests}

The authors declare that they have no competing interests.

\section{Author details}

${ }^{1}$ Western Seoul Center, Korea Basic Science Institute, Seoul, South Korea. Department of Environmental Science and Engineering, Ewha Womans University, Seoul, South Korea. ${ }^{3}$ Seoul Center, Korea Basic Science Institute, Seoul, South Korea. ${ }^{4}$ Department of Chemical Engineering and Materials Science, Ewha Womans University, Seoul, South Korea. 
Received: 12 April 2016 Accepted: 8 July 2016

Published online: 19 July 2016

\section{References}

Beck HC, Hansen AM, Lauritsen FR. Novel pyrazine metabolites found in polymyxin biosynthesis by Paenibacillus polymyxa. FEMS Microbiol Lett. 2003; 220:67-73.

Bergknut M, Frech K, Andersson PL, Haglund P, Tysklind M. Characterization and classification of complex PAH samples using GC-qMS and GC-TOFMS. Chemosphere. 2006;65:2208-15.

Choi NR, Kim YP, Ji WH, Hwang G-S, Ahn YG. Identification and quantification of seven volatile $n$-nitrosamines in cosmetics using gas chromatography/ chemical ionization-mass spectrometry coupled with head space-solid phase microextraction. Talanta. 2016;148:69-74.

Clery RA, Hammond CJ, Wright AC. Nitrogen-containing compounds in black pepper oil (Piper nigrum L.). J Essent Oil Res. 2006;18:1-3.

de Oliveira AM, Pereira NR, Marsaioli A, Augusto F. Studies on the aroma of cupuassu liquor by headspace solid-phase microextraction and gas chromatography. J Chromatogr A. 2004;1025:115-24.

Dickschat JS, Wickel S, Bolten CJ, Nawrath T, Schulz S, Wittmann C. Pyrazine biosynthesis in Corynebacterium glutamicum. Eur J Org Chem. 2010;2010: 2687-95.

Fang M, Cadwallader KR. Convenient synthesis of stable deuterium-labeled alkylpyrazines for use in stable isotope dilution assays. J Agric Food Chem. 2013;61:3580-8

Grebel JE, Young CC, Suffet IH. Solid-phase microextraction of N-nitrosamines. J Chromatogr A. 2006;1117:11-8.

Harris DC. Quantitative chemical analysis. New York: W. H. Freeman and Co. 2010.

Harris R, Lacey M, Brown W, Allen M. Determination of 2-methoxy-3alkylpyrazines in wine by gas chromatography/mass spectrometry. Vitis. 1987; 26:201-7.

Hwang H-I, Hartman TG, Rosen RT, Lech J, Ho C-T. Formation of pyrazines from the Maillard reaction of glucose and lysine-alpha-amine-15N. J Agric Food Chem. 1994;42:1000-4.

Koehler PE, Mason ME, Newell JA. Formation of pyrazine compounds in sugaramino acid model systems. J Agric Food Chem. 1969:17:393-6.

Muller R, Rappert S. Pyrazines: occurrence, formation and biodegradation. Appl Microbiol Biotechnol. 2010;85:1315-20.

Nawrath T, Dickschat JS, Kunze B, Schulz S. The biosynthesis of branched dialkylpyrazines in myxobacteria. Chem Biodivers. 2010;7:2129-44

Pawliszyn J. Theory of solid-phase microextraction. J Chromatogr Sci. 2000;38: 270-8.

Rajini KS, Aparna P, Sasikala C, Ramana CV. Microbial metabolism of pyrazines. Crit Rev Microbiol. 2011;37:99-112

Risticevic S, Lord H, Gorecki T, Arthur CL, Pawliszyn J. Protocol for solid-phase microextraction method development. Nat Protoc. 2010;5:122-39.

Rizzi GP. Formation of pyrazines from acyloin precursors under mild conditions. J Agric Food Chem. 1988;36:349-52.

Sala C, Mestres M, Martı M, Busto O, Guasch J. Headspace solid-phase microextraction analysis of 3-alkyl-2-methoxypyrazines in wines. J Chromatogr A. 2002;953:1-6.

Schulz S, Dickschat JS. Bacterial volatiles: the smell of small organisms. Nat Prod Rep. 2007;24:814-42.

Shin SC, Kim SH, You H, Kim B, Kim AC, Lee KA, Yoon JH, Ryu JH, Lee WJ, Drosophila microbiome modulates host developmental and metabolic homeostasis via insulin signaling. Science. 2011;334:670-4.

Shu C-K. Pyrazine formation from serine and threonine. J Agric Food Chem. 1999; 47:4332-5.

Theodoridis G, Koster EH, De Jong G. Solid-phase microextraction for the analysis of biological samples. J Chromatogr B Biomed Sci Appl. 2000;745:49-82.

Wampler TP. Analysis of food volatiles using headspace-gas chromatographic techniques. Techniques for analyzing food aroma. 1997. p. 27-58.

Yang X, Peppard T. Solid-phase microextraction for flavor analysis. J Agric Food Chem. 1994:42:1925-30.

Zhang Z, Pawliszyn J. Headspace solid-phase microextraction. Anal Chem. 1993; 65:1843-52.

\section{Submit your manuscript to a SpringerOpen ${ }^{\circ}$ journal and benefit from:}

- Convenient online submission

- Rigorous peer review

- Immediate publication on acceptance

- Open access: articles freely available online

- High visibility within the field

- Retaining the copyright to your article

Submit your next manuscript at $\boldsymbol{s p r i n g e r o p e n . c o m ~}$ 